\title{
An Optimal Text/Background Color Combination of LED Information Boards for Visibility Improvement Based on Psychological Measurements
}

\author{
Aya Shiraiwa $^{1}$, Eriko Aiba ${ }^{1,2,3}$, Takayuki Shimotomai ${ }^{4}$ Hiroya Inome $^{1}$, \\ and Noriko Nagata ${ }^{1}$ \\ ${ }^{1}$ Graduate School of Science and Technology, Kwansei Gakuin University, 2-1 \\ Gakuen, Sanda, Hyogo 669-1337, Japan \\ \{shiraiwa.aya, nagata\}@kwansei.ac.jp \\ 2 National Institute of Advanced Industrial Science and Technology, 1-8-31 \\ Midorigaoka, Ikeda, Osaka 563-8577, Japan \\ 3 Research Fellow of the Japan Society for the Promotion of Science \\ aiba.eriko@aist.go.jp \\ 4 Brain Science Institute, Tamagawa University, 6-1-1 Tamagawa-gakuen, Machida, \\ Tokyo 194-8610, Japan \\ shimotomai@lab.tamagawa.ac.jp
}

\begin{abstract}
In this study, conspicuity and readability of the text on light-emitting diode (LED) information boards were evaluated by psychological experiments, and the influences of color combinations of text / background, luminance, and shadow were evaluated quantitatively. In recent years, LED boards have been able to display full-color images, and they have many functions to highlight text. Therefore, it is assumed that the LED board ability to function may be reduced depending on the way it is used. We investigated the relationship of color combinations of text and background, luminance, and shadow to conspicuity and readability to evaluate ease and efficiency of information communication via the LED board. We found that (1) the size of the area with high luminance affected conspicuity, (2) the difference of luminance of the part adjacent to the text/background affected readability, and (3) shadow affected both conspicuity and readability. Furthermore, trade-off between them was confirmed.
\end{abstract}

Keywords: visibility, LED, color combination, Scheffe's paired comparison.

\section{Introduction}

In recent years, not only for lighting and television, but also for electronic displays such as various traffic signs and displays, the light-emitting diode (LED) has been well-used. In addition to its major characteristics, such as compact

S. Tominaga, R. Schettini, and A. Trémeau (Eds.): CCIW 2013, LNCS 7786, pp. 119-132, 2013.

(C) Springer-Verlag Berlin Heidelberg 2013 
size, energy savings, and long life, LED ability to display full color and high luminance intensity has improved, so the LED is being used for various purposes. LEDs have been used widely, especially as electronic displays, because of their ability to display in full color including white color, show blinking or scrolling text, and display text or pictures easily.

The object of this study is the information indicator LED board in which LEDs are placed in a dot-matrix configuration, such as the LED traffic sign. We investigated the visibility of the LED boards.

Visibility is defined as the degree of conspicuousness of the objects [1], 2], [3. When information is displayed by using LED boards, not only the LED board itself needs to be noticeable, but the contents also need to be understood. Moreover, because LED boards have various functions, it is possible to reduce the original LED boardfs ability to convey information depending on the way it is used. Therefore, the relationship of the optimal color combination of text and background, luminance intensity, and shadow needs to be arranged properly so that LED board users are able to send information easily and efficiently.

Regarding conspicuity and readability of the color combination of text and background, many previous studies have been conducted using a cathode ray tube (CRT) or liquid crystal display (LCD) [4], [5], 6], [7]. Moreover, there are various studies; such as the impact of web page text/background color combinations on retention of memory and readability and the effects of text/background color combination on readability of electric paper and LCD in discriminating performance for young and elderly [3], 8], [9]. The one previous study using LEDs examined the effect of the color combination of text/background on human comfort and readability with LED display computers [10. However, there is no study concerning LED boards on which LEDs are placed in a dot-matrix configuration, such as the LED traffic sign. Furthermore, even though shadow text is often used to emphasize the text or sentence on LCD displays, there are only a few studies considering conspicuity and readability with shadow text. Considering previous studies, not using CRT, LCD, or LED display computers, but using LED boards, there is a possibility that characteristic effects of LED on color combinations of text and background may appear.

Therefore, the purpose of this study is to quantify the factor of visibility of color combinations of text/background on LED boards, that is, conspicuity and readability.

In this study, we used LED boards and changed color combinations of text and background each time. Then, we evaluated the visibility based on a paired comparison. At first, we used chromatic color and arranged the experiment considering luminance; then evaluation of conspicuity and readability was conducted by a psychological scale. Next, the experiment to add achromatic colored shadow to the text was conducted, and we evaluated the influence of the stereoscopic effect added by shadow text for conspicuity and readability. Furthermore, from the findings of Experiment 1, we evaluated visibility when difference of luminance of chromatic color was significant, not considering the luminance. Lastly, integrating the result of these experiments, it was determined that conspicuity 
and readability have a trade-off relationship in color combinations of text and background. It was also determined that visibility was improved by difference of luminance or by adding shadow on the text.

\section{Experiment 1: The Influence of the Hue under the Condition of Same Luminance}

In Experiment 1, we investigated the influence of the difference of the hue on conspicuity and readability, under the condition of same luminance. Previous studies have demonstrated that the difference between the colors of text and background affects conspicuity and readability. However, various opinions about the influence of the hue on the color combination have not yet been confirmed. Therefore, in Experiment 1, the effect of hue under the condition of same luminance was investigated.

\subsection{Method}

Participants. 116 observers (97 male and 19 female; average age: 20.9 years old) participated in Experiment 1.

Apparatus. The text stimuli were shown on the information indicator (LED board) with LEDs placed as a dot-matrix display. The LED board was placed positioned $180 \mathrm{~cm}$ from the floor. The experiment was arranged in the lecture room at Kwansei Gakuin University. The participants took seats between nine lines of right and left and 13 lines of anteroposterior. Each participant's seat location was recorded. The LED board, except for the area of stimuli indication, was covered with a black cloth. The luminous intensity of the LED board was 905 lux, under the condition with lights on and curtains of the lecture room closed.

Stimuli. We used the text letter "E" as the stimulus, displayed in red, green, blue, and yellow. The colors of the background were red, green, blue, yellow, black, and white. Twenty combinations of text and background color were used. The red, green, and yellow used in the experiment were not pure color, because each luminance of the color was standardized based on the luminance of blue, which was the darkest luminance among of them.

Procedure. We showed four stimuli of "E" with different color combinations of texts and backgrounds at the same time on an LED board. Exposure duration for the stimuli was 15 seconds at five-second intervals, with each exposure duration and interval counted as one trial; we repeated the stimulus for 78 trials. Furthermore, we considered the influence of the luminance by using black and white, which were of a different luminous intensity from the chromatic color. From the first trial to the 65th trial, all patterns of color combination stimuli were shown at random, then, from the 66 th trial to 78 th trial, we only showed the color combination stimuli that did not include black and white. 
Task. Regarding conspicuity and readability of stimuli presented to the participants, they were instructed to indicate a ranking of readability from No. 1 to No. 4 on a questionnaire for each trial.

Analysis. The data were analyzed using Scheffe's paired comparison method (modified Haga's method). Participants arranged the ranking for four patterns of stimuli that were presented to participants at the same time. We made these four rankings paired, two stimuli each. Then, we analyzed as a total of six pairs. Although the correlation with these results and the seat location was also verifies, it was confirmed that the seat location did not affect the results.

\section{$2.2 \quad$ Results}

The results of Experiment 1 are shown in Fig. 1 and 2 Fig. 1 indicated the conspicuity of stimuli patterns, and Fig. 22 was for readability of stimuli patterns. The vertical axis indicates the evaluation value for conspicuity and readability (z-score; gap value from the average value for each data), and the horizontal axis showed that a negative value indicated a better evaluation.

From the results of Experiment 1, the characteristic results were obtained concerning both conspicuity and readability for the color combination stimuli patterns with the black/white backgrounds. For conspicuity, all patterns with a white background obtained higher ratings. Almost all patterns with a black background obtained a lower rating. Besides the white background, the red tended to receive a better rating. For readability, the stimuli patterns with black or white background tended to receive higher rating. We could not find a particular tendency for the color combination patterns combining chromatic colors. Furthermore, there was no major difference between the 66th trial and the 78th trial, which tested only color combination patterns without black and white being a different luminance from the chromatic color. Therefore, we were not able to confirm the influence of the difference of hue (combination of chromatic colors).

\section{Experiment 2: The Influence of Shadow Text}

We investigated the effect of shadow text, which increases the stereoscopic effect. We investigated whether or not this stereoscopic effect affects conspicuity and readability. Contrary to Experiment 1, we used only achromatic color in this experiment so that we could focus on only the difference of luminance to investigate conspicuity and readability in this experiment. Furthermore, we considered the stereoscopic effect obtained by the luminance of the color of the shadows, or by existence or non-existence of the shadow.

\subsection{Method}

Participants. 18 observers (15 male and three female; average age: 22.2 years old) participated in Experiment 2. 


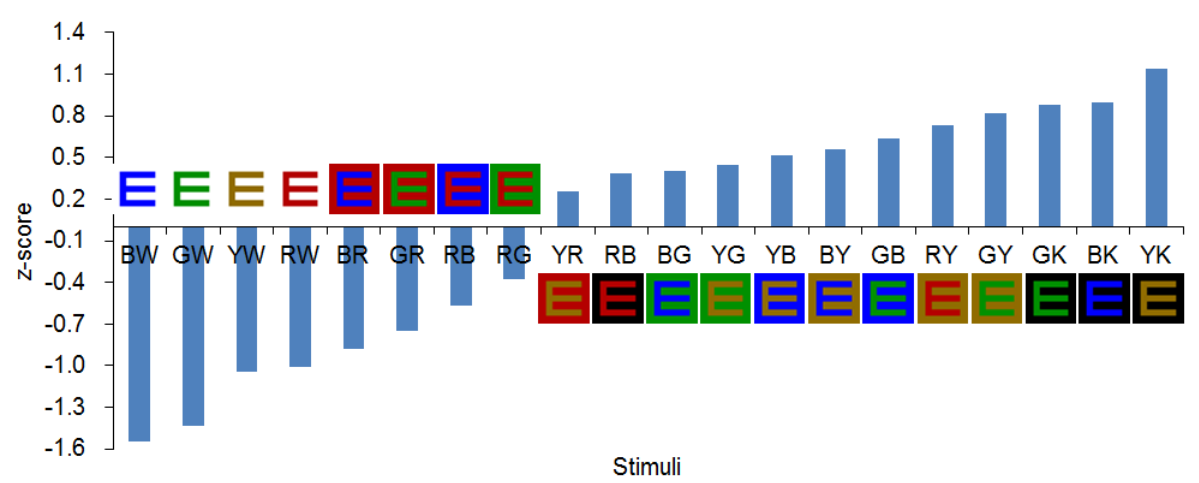

Fig. 1. Psychological measure for conspicuity under the condition of same luminance: First letter is text color and second letter is background color. R, Red; G, Green; B, Blue; Y, Yellow; K, Black; W, White.

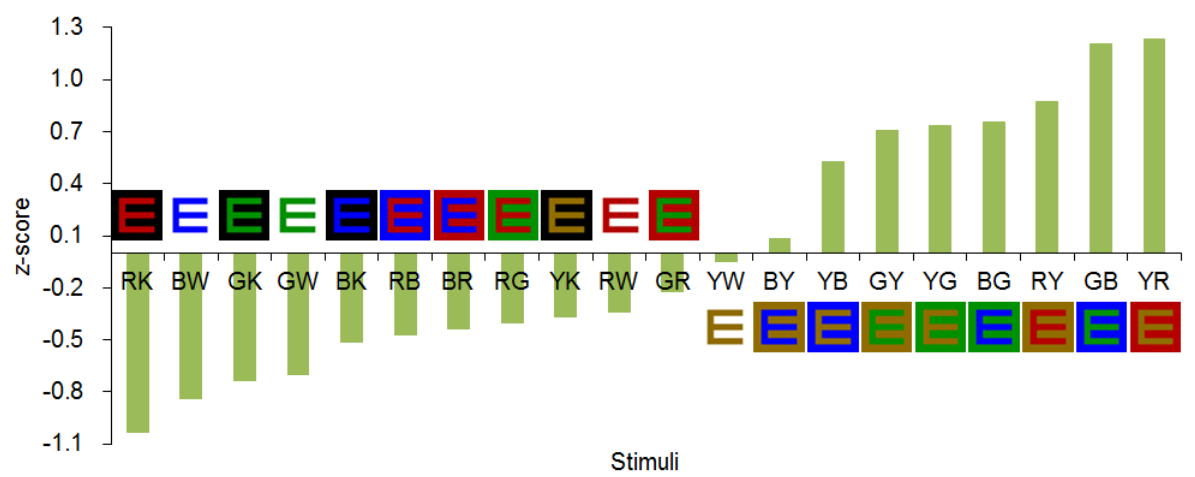

Fig. 2. Psychological measure for readability under the condition of same luminance: First letter is text color and second letter is background color. R, Red; G, Green; B, Blue; Y, Yellow; K, Black; W, White.

Apparatus. The apparatus is the same as in Experiment 1.

Stimuli. Stimuli were presented on an LED board in the same manner as Experiment 1. The text of gEh was presented as in Experiment 1. The colors of the text and the background were black, white, and gray. The colors of the shadow were black, white, and gray. There were two patterns of stimuli presented: "with shadow" and "without shadow." The 12 patterns of stimuli presented used combinations of different colors for text, background, and shadow.

Procedure. We presented two patterns of stimuli at the same time. Exposure duration and intervals of the stimuli were the same as the procedure of 
Experiment 1. Participants observed stimuli during 66 trials. A trial consisted of a 15-second exposure duration and a five-second interval.

Task. Concerning three items - "conspicuity," "readability," and "stereoscopic effect" of indicated stimuli - the participants were instructed to choose the better one from each presented paired of stimuli, and to fill out a six-step evaluation for each presented pair in a questionnaire.

Analysis. As in Experiment 1, the data were analyzed using Scheffe's paired comparison method (modified Haga's method).

\subsection{Results}

The results of Experiment 2 are shown in Fig. 3, 4, and 5] Fig. 3] indicates the conspicuity of stimuli patterns, Fig. 4 shows readability of stimuli patterns, and Fig. 5 shows stereoscopic effect. The vertical axes of Fig. 3- 5 were for evaluation value of conspicuity, readability, and stereoscopic effect (z-score). The horizontal axis indicated color combination patterns of presented stimuli. In the vertical axis a negative value indicates a better evaluation.

For conspicuity, as in the results of Experiment 1, the white background obtained the highest evaluation. Color combination patterns in order of obtaining high evaluation ranked 1) white background, 2) white text, and 3) white shadow text. Color combination patterns without any white received the lowest evaluation rating. For readability, larger differences between color of text and background or between luminance of color of text and shadow (white and black) obtained higher evaluations. Concerning the stereoscopic effect, the black shadow received the highest evaluation. Poor evaluation was given to text without shadow.

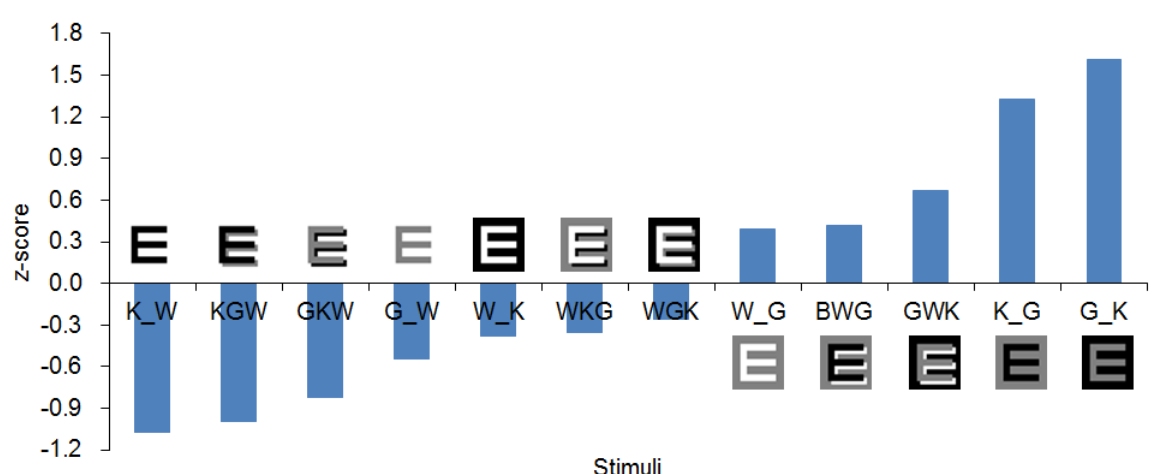

Fig. 3. Psychological measure for conspicuity under the condition of shadow text: First letter is text color and last letter is background color. Second letter is shadow color and "." is without shadow. K, Black; W, White; G, Gray. 


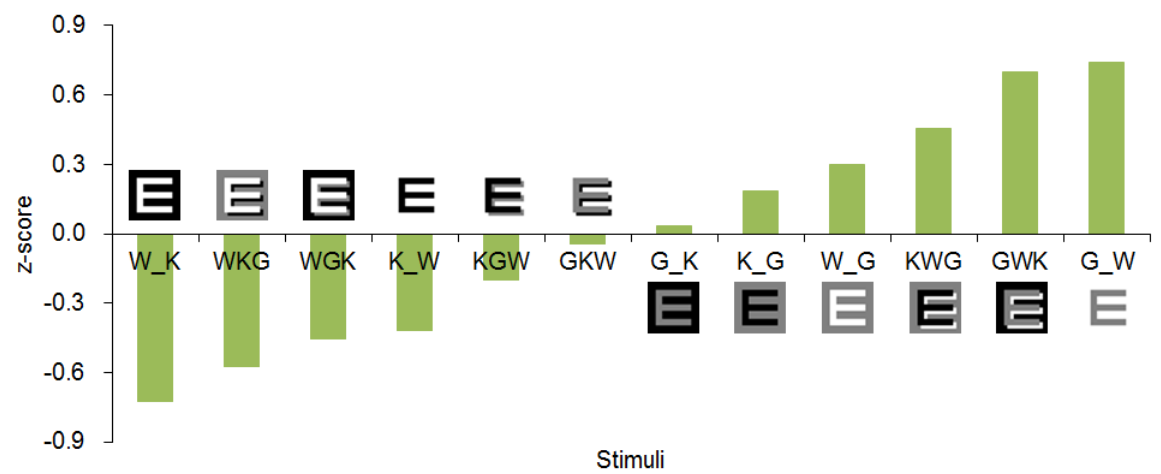

Fig. 4. Psychological measure for readability under the condition of shadow text: First letter is text color and last letter is background color. Second letter is shadow color and "." is without shadow. K, Black; W, White; G, Gray.

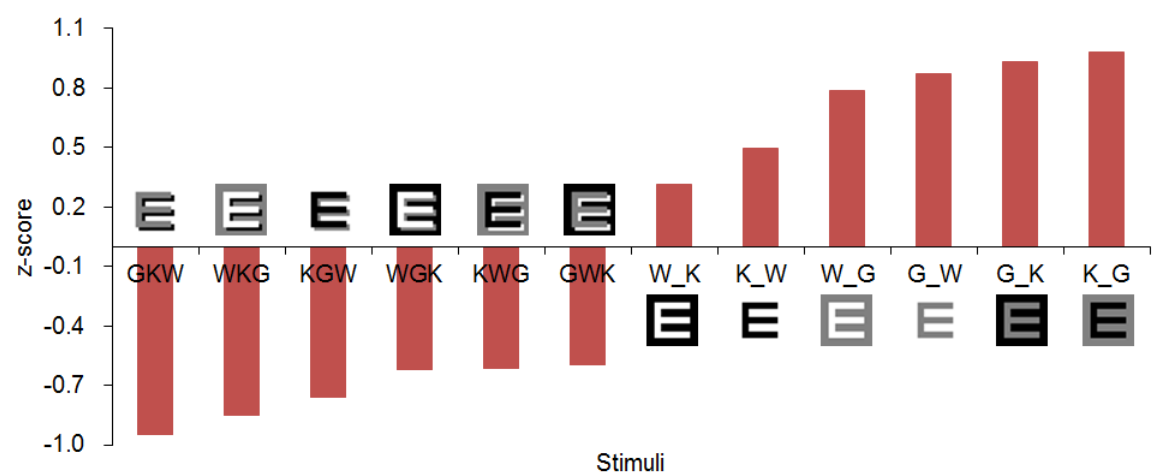

Fig. 5. Psychological measure for stereoscopic effect under the condition of shadow text: First letter is text color and last letter is background color. Second letter is shadow color and "." is without shadow. K, Black; W, White; G, Gray.

\section{Experiment 3: Influence of Hue without Respect to Luminance}

In Experiment 3, unlike Experiment 1 in which the luminance of color of text and background were arranged the same, we investigated the color combination patterns with high evaluations by disregarding the difference in luminance and using pure colors. Furthermore, we examined conspicuous and readable color combination patterns.

\subsection{Method}

Participants. 64 observers (54 male and 10 female; average age: 19.4 years old) participated in Experiment 3. 
Apparatus. The apparatus is the same as in Experiment 1.

Stimuli. As in Experiment 1, we presented stimuli on an LED board. The text of "E" was presented as in Experiment 1. To arrange the difference of luminance for the largest possible contrast, we divided the colors into groups of high luminance and low luminance. The group of high luminance included white and yellow, and the group of low luminance included black, blue, and red. The text and background color were from the two different luminance groups. A total of 12 patterns of stimuli were presented. Unlike Experiment 1, we used pure colors only in Experiment 3.

Procedure. The procedure is the same as in Experiment 2.

Task. For two items, "conspicuity" and "readability" of presented stimuli, the participants were instructed to choose the better one from the presented paired of stimuli, then to fill out a six-step evaluation for each pair in questionnaire.

Analysis. The analysis is the same as in Experiment 2.

\subsection{Results}

The results of Experiment 3 are shown in Fig. 6] and 7. Fig. 6] indicates the conspicuity of stimuli patterns, and Fig. 7 indicates readability of stimuli patterns. The vertical axes of Fig. 6 and 7 were for evaluation value of conspicuity and readability (z-score), and the horizontal axis indicated color combination patterns of presented stimuli. In the vertical axis a negative value indicated the better evaluation.

For conspicuity, stimuli patterns using red obtained high evaluation rating, and stimuli patterns using black received low evaluation. Stimuli patterns of white-colored background obtained high evaluations in Experiment 1 and 2; however, in this experiment, its evaluation changed based on colors of the text. Conversely, from the view of readability, stimuli patterns using black or blue received high evaluation. On the other hand, stimuli patterns using red received low evaluation.

In addition, the evaluation rate of readability added to the evaluation rate of conspicuity made the value of another characteristic we defined as "visibility," that is, whether we could see the visual objects clearly or not. Fig. 8 indicates the value of visibility. The vertical axis and the horizontal axis of Fig. 8 were the same as Fig. 6] and 7 For visibility, stimuli patterns using blue obtained high evaluation ratings, and stimuli patterns using black received low evaluations. Then, analyzing Fig. 8 , it was found that stimuli patterns that were highly evaluated tended to be lined up in order of replacing color of text and background. Therefore, the evaluation rate of visibility tended to be rather fixed by color combination.

In Experiment 2, the color combination stimuli pattern with Black and White which had the biggest difference of luminance obtained good evaluation for readability especially. However, in this experiment, there was the color combination 
stimuli pattern obtained better evaluation than Black and White stimuli pattern of Experiment 2. Therefore, besides difference of luminance, there could be existed the parameter which affects readability. According to previous study [1], it has been shown that psychological measure for "favorability" on color combination affects readability. This was also suggested by the result that the value of visibility was lined up in order of each pattern of color combinations.

\section{Experiment 4: The Influence of Color Combination and Shadow Text}

In Experiment 4, based on the results from Experiments 1 to 3, we investigated which pattern of color combination was compatible with high conspicuity and readability among the group of color combination patterns of text and background including shadow text.

\subsection{Method}

Participants. 95 observers (73 male and 22 female; average age: 20.7 years old) participated in Experiment 4.

Apparatus. The apparatus is the same as in Experiment 1.

Stimuli. As in Experiment 1, we presented stimuli on an LED board. The text of "E" was presented just like Experiment 1. The text colors were white and yellow; the background colors were blue and red. Each combination was arranged with or without shadow text (total eight patterns). In addition, total four patterns were used: black text on white background and white text on black background

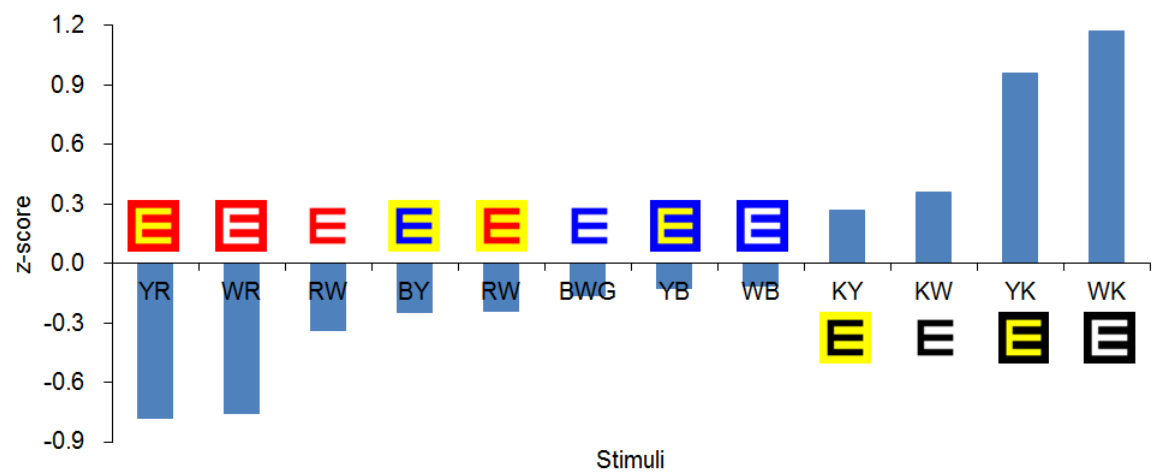

Fig. 6. Psychological measure for conspicuity under the condition without respect to luminance: First letter is text color and second letter is background color. R, Red; B, Blue; Y, Yellow; K, Black; W, White. 


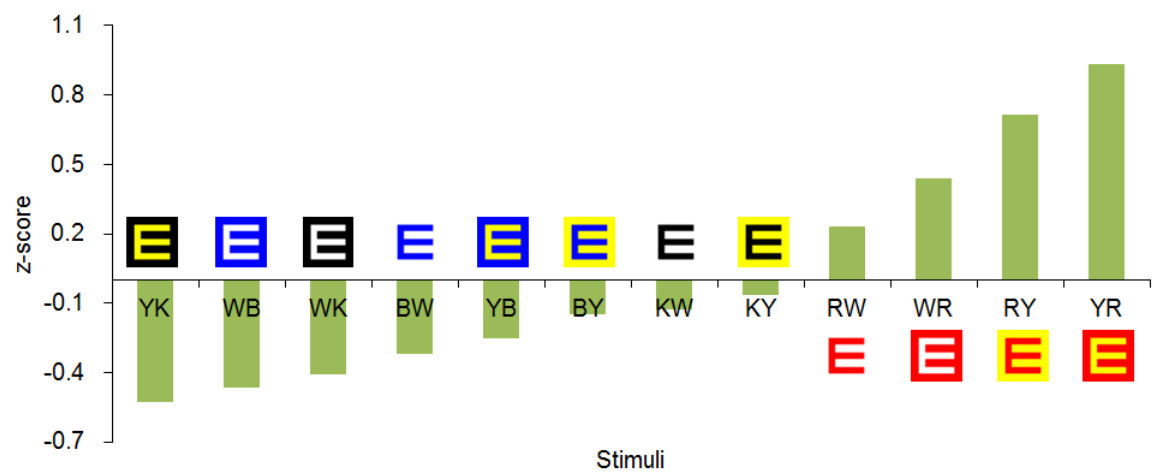

Fig. 7. Psychological measure for readability under the condition without respect to luminance: First letter is text color and second letter is background color. R, Red; B, Blue; Y, Yellow; K, Black; W, White.

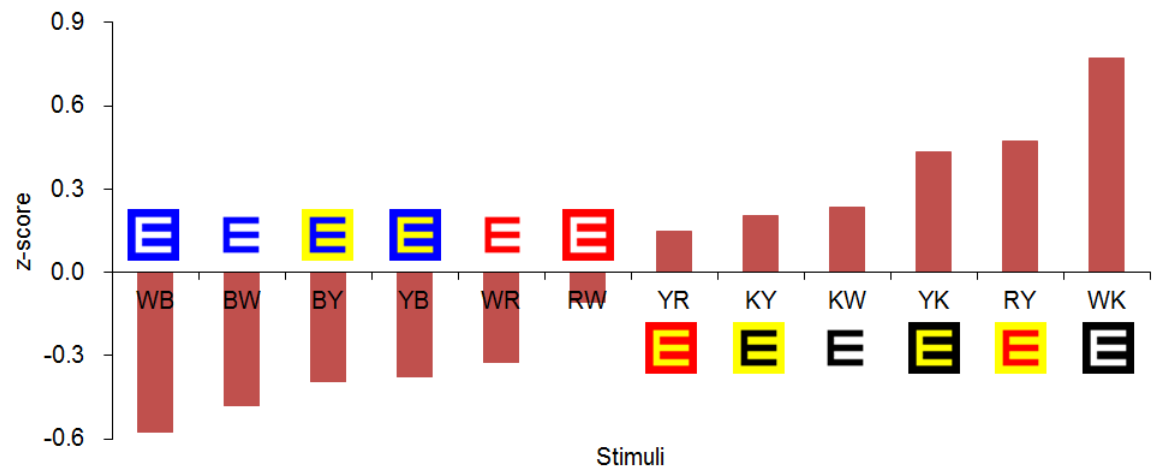

Fig. 8. Psychological measure for visibility under the condition without respect to luminance: First letter is text color and second letter is background color. R, Red; B, Blue; Y, Yellow; K, Black; W, White.

as the index of the difference of luminance; white text on gray background with black shadow, which received a high evaluation in Experiment 2; and white text on yellow background with black shadow, which has high luminance for both color of text and background. Total of stimuli patterns was 12 patterns.

Procedure. The procedure is the same as in Experiment 2.

Task. Concerning two items, "conspicuity" and "readability" of indicated stimuli, the participants were instructed to choose the better one from the presented paired of stimuli, then to fill out a six-step evaluation for each pair in the questionnaire.

Analysis. The analysis is the same as in Experiment 2. 


\subsection{Results}

The results of Experiment 4 are shown in Fig. 9 and 10, Fig. 9 indicated the conspicuity of stimuli patterns, and Fig. 10was for readability of stimuli patterns. The vertical axes of Fig. 9 and 10 measure the evaluation value of conspicuity and readability (z-score), and the horizontal axis indicates color combination patterns of presented stimuli. In the vertical axis a negative value indicates the better evaluation.

Matching the results of Experiment 3, combinations with a red background were conspicuous but hard to read and the patterns with a black or blue background were easy to read and conspicuous. It was also found that the pattern of white text/yellow background/black shadow with highest luminance of text and background was most conspicuous but the hardest to read. Furthermore, by conducting Experiment 4, we found that the evaluations for both conspicuity and readability would be higher when shadow was added to the text.

Regarding conspicuity, significant differences at p .01 were found between text and background in the following paired patterns: white text on yellow background with shadow and yellow text on red background with shadow; yellow on red with shadow and white on red; white on red with shadow and white on red; white on red and white on blue with shadow; yellow on red and black on white; white on blue with shadow and yellow on blue; black on white and white on gray with shadow; yellow on blue and white on gray with shadow; white on blue and white on gray with shadow; white on gray with shadow and white on black. Then, significant differences of the level of $\mathrm{p} .05$ were found in the following paired patterns: yellow on blue with shadow and white on blue with shadow.

Regarding readability, significant differences at the level of $\mathrm{p} .01$ were found in the following paired patterns: yellow text on blue background with shadow and white text on blue background with shadow; white on blue with shadow and white on blue; white on black and white on blue; white on red with shadow and white on blue; white on blue and yellow on red with shadow; black on white and white on red; yellow on blue and white on red; white on gray with shadow and white on red; white on red and yellow on red; yellow on red and white on yellow with shadow.

Furthermore, the pattern of white text on red background with black shadow marked the highest visibility, which was defined as the sum of conspicuity and readability. We determined that this pattern used the background with conspicuous warm colors and readable white text and black shadow so this pattern was compatible with conspicuity and readability.

\section{Discussion}

We investigated the effect of shadow text and the color combination that would improve conspicuity and readability of presented texts on an LED board. Here, we discuss the effect influenced by color combination or shadow text.

First, it was determined that the pattern would be more conspicuous by making the area with high luminance (white or yellow) wider, or by using a warm color (red). Moreover, it was also determined that the pattern would be more 


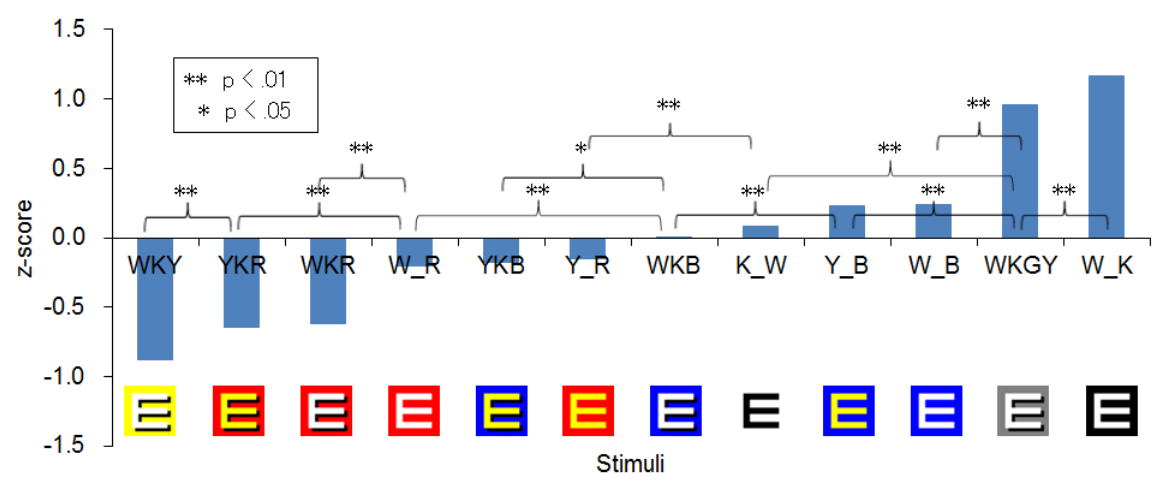

Fig. 9. Psychological measure for conspicuity under the condition of considering color combination and shadow text: First letter is text color and last letter is background color. Second letter is shadow color and "_" is without shadow. R, Red; B, Blue; Y, Yellow; K, Black; W, White; GY, Gray.

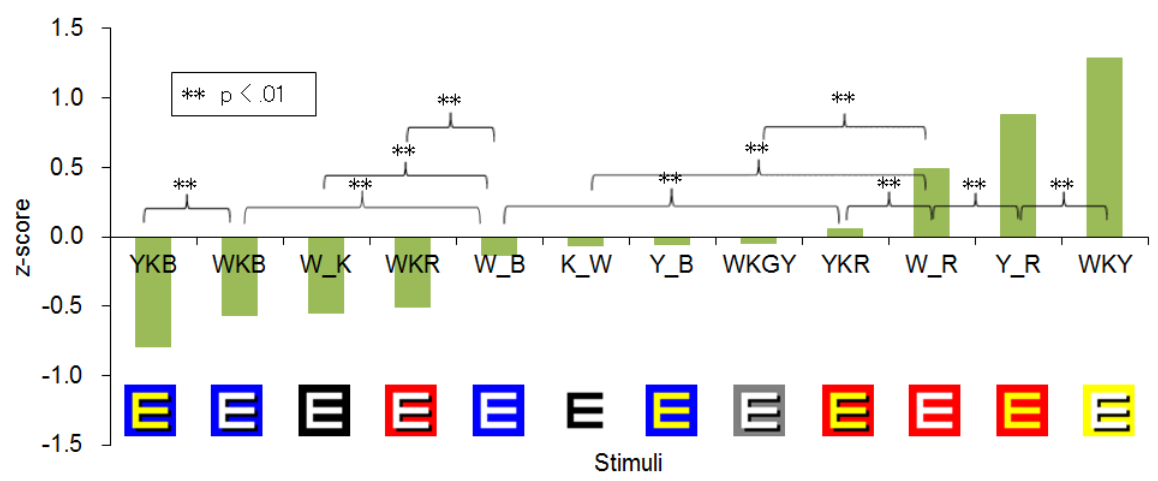

Fig. 10. Psychological measure for readability under the condition of considering color combination and shadow text: First letter is text color and last letter is background color. Second letter is shadow color and "_" is without shadow. R, Red; B, Blue; Y, Yellow; K, Black; W, White; GY, Gray.

readable by making the difference of luminance between color of text and background bigger, or by using a blue or black background. From these, a "trade-off" relationship between conspicuity and readability was seen. That is, it could be not as conspicuous for a color combination that feels good when reading the text, but if we do not feel good when reading, it could be conspicuous.

In Experiment 1, the luminance of the chromatic color was standardized and pure color was not used. In Experiment 1, characteristic results for conspicuity were found in the stimuli patterns using black and white, which had a difference of luminance compared with chromatic color. However, in Experiment 3 that luminance was disregarded and pure color was used; the tendency shown in the results of Experiment 1 was not repeated. From these facts, we found out that an 
influence stronger than the difference of luminance could be obtained by using pure color with the highest saturation in each hue.

Moreover, it was also determined that adding black shadow to the text affected conspicuity and readability. Regarding the color of the shadow, black gave the strongest stereoscopic effect. Furthermore, we received some reports from the participants that they felt text with gray or white shadow was unnatural because such colors of shadow do not exist in nature. Therefore, it was assumed that shadow text with a color that exists in nature should be more effective in accomplishing the stereoscopic effect. Color combination was a "trade-off" relationship between conspicuity and readability, so it was assumed that the stimuli pattern with an unnatural shadow color could be conspicuous but not readable. However, it was actually the case that this pattern received low evaluation for both conspicuity and readability. Based on this fact, to make the luminance of the part adjacent to the text more highlighted, we assumed that using shadow text would achieve this most effectively only if a natural color pattern were used.

In this study, we used combinations of different colors for text and background; however, previous studies determined that there was a color that was most suitable for texts [11]. Future studies should address how these findings would relate to people's color perception or visibility.

\section{Conclusion}

We conducted four experiments in this study for the purpose of improving conspicuity and readability of color combinations of text and background on an LED board. For conspicuity, it was confirmed that the pattern would be more conspicuous by making the area with high luminance wider, or by using a warm color. For readability, it was confirmed that the pattern could be more readable by increasing the difference of luminance between the part adjacent to the text and the background, or by using a particular color combination. Furthermore, by adding black shadow to the text, it was confirmed that both conspicuity and readability were improved. Then, a "trade-off" relationship between conspicuity and readability was suggested.

We evaluated visibility, that is, conspicuity and readability, of the text on an LED board from the view of color combination. However, many other parameters affect visibility. In future, research should examine the difference of visibility depending on where the LED board is placed, the luminance of the LED board, and the difference in visibility created by the text font.

\section{References}

1. American National Standard for Human Factors Engineering of Visual Display Terminal Workstations. Human Factors and Ergonomics Society, Santa Monica, LA (1988)

2. Glossary of Technical Terms in Japanese Industrial Standards 5th Edition. Japanese Standards Association, Tokyo (2001) (in Japanese) 
3. Gradisar, M., Humar, I., Turk, T.: The Legibility of Colored Web Page Texts. In: ITI 2007 29th Int. Conf. on Information Technology Interfaces (2007)

4. Shieh, K., Lin, C.: Effects of Screen Type, Ambient Illumination, and Color Combination on VDT Visual Performance and Subjective Preference. International Journal of Industrial Ergonomics 26, 527-536 (2000)

5. Lin, C.-C.: Effects of Contrast Ratio and Text Color on Visual Performance with TFT-LCD. International Journal of Industrial Ergonomics 31, 65-72 (2003)

6. Lin, C.-C.: Effects of Screen Luminance Combination and Text Color on Visual Performance with TFT-LCD. International Journal of Industrial Ergonomics 35, 229-235 (2005)

7. Hall, R.H., Hanna, P.: The Impact of Web Page Text-background Colour Combinations on Readability, Retention, Aesthetics and Behavioural Intention. Behaviour \& Information Technology 23, 183-195 (2004)

8. Humar, I., Gradisar, M., Turk, T.: The Impact of Color Combinations on the Legibility of a Web Page Text Presented on CRT Displays. International Journal of Industrial Ergonomics 38, 885-899 (2008)

9. Wang, A., Hwang, S., Kuo, H.: Effects of Text/background Color Combination, ambient Illuminance, and Display Type on Discriminating Performance for Young and Elderly Users. Journal of the Society for Information Display 20, 87-93 (2012)

10. Lee, E., Suk, H.: The Effect of Color Contrast between Text and Background on Human Comfort - Psychological and Physiological Investigations. Midterm Meeting of the International Colour Association (AIC 2011) (2011)

11. Saburi, S.: Structure of Visibility for the Combination of Background and Character Colors - an Analysis by a Maximum Likelihood Asymmetric Multidimensional Scaling. Japanese Journal of Behaviormetrics 35, 193-201 (2008) (in Japanese) 